\title{
Investigation of friction mechanisms in finger pad sliding against surfaces of varying roughness
}

\author{
G.P. Chimata and C.J. Schwartz* \\ Department of Mechanical Engineering
}

lowa State University, Ames, lowa, USA

\begin{abstract}
Human finger pad friction and the effects of surface features on the fingerprint ridges, shape of the finger and the material properties of the finger were investigated. The friction coefficients for three fine-grit abrasive papers were measured to assess these effects. Four probing surfaces were used: human index finger pad, silicone replicas of the finger with and without fingerprints, and a smooth silicone sphere. Friction tests were performed at a constant normal load of $0.5 \mathrm{~N}$ in two probe orientations: normal and perpendicular to the orientation of the fingerprint ridges. Scanning electron microscopy of the abrasive papers was performed to examine their surface topography. Based on the trends in coefficients of friction, topography of the abrasive papers, surface features of the finger pad, and the shape and elastic properties of the finger, possible friction mechanisms were discussed. It was inferred that the change in the shape of the probe (from a sphere to the finger shape) changes the adhesion and deformation components of friction, presence of fingerprints adds an interlocking contribution to the friction and decrease the adhesion and deformation components of friction, and the elastic properties of the finger lead to an increase in all the three components of friction.
\end{abstract}

Keywords: skin tribology, finger pad friction, friction mechanisms, fingerprints, tactility, haptics. 


\section{Introduction}

Fingers play an important role in handling, manipulating, gripping and perceiving surfaces and objects during multiple everyday tasks. It was established that the friction between the fingers and an object is one of the factors that influences the grip force employed by individuals in precision grip activities like grasping and lifting objects[1]. During active tactile exploration of surfaces using fingertips, it was observed that the normal contact force and tangential shear force (friction force) were optimally adjusted depending on the topography of the surface being explored[2]. Therefore, it’s evident that finger-surface friction is important for controlling all these interactions, and understanding the factors affecting finger pad friction can help in designing surfaces for improved efficiency in gripping, manipulation and perception tasks.

The coefficient of friction of a finger pad is not an intrinsic property of the skin and is dependent on the tribological system for which the measurement was made. The contact mechanics is influenced by the material and microgeometry of both the surfaces in contact, skin hydration, normal load and the relative velocity of the surfaces in contact. Finger pad friction properties were measured against a wide range of materials so far such as packaging materials[3], textiles[4, 5],metals[6-8], polymers[6, 8] and glass[9, 10] to name a few. The effect of the interacting surface's microgeometry on finger friction has been explored in studies on ridged surfaces with different ridge geometries to understand their effect on finger friction[7, 11]. Finger pad friction was used for comparing materials with skin like properties [4], designing artificial fingers[12, 13] and tactile attributes of textiles[5]. The effect of applied variables like normal force, sliding velocity and lubricants on finger pad friction has also been well characterized [6, 8, 14]. Inherent skin related phenomena like occlusion, sweating and the sensitivity of coefficient of friction to these conditions were well investigated[9, 10]. More 
recently, Derler et al. identified ploughing and abrasion as important friction mechanisms during repetitive rubbing of fingerpad on abrasive surfaces[15].

Even with the existing research, the effects of surface features and the topography of the finger pad on the friction are not well characterized. Sliding a finger and a finger like sensor with ridges mimicking fingerprints across randomly textured surfaces induced oscillations in friction force of frequency that can be optimally received by the skin mechanoreceptors [16-18]. Further, it has been suggested previously that fingerprint ridges play a role in determining friction when interacting with ridged surfaces of dimensions greater than fingerprint ridges [7]. However, the effect of fingerprints on friction when in sliding contact with surface features much smaller than fingerprint ridge dimensions is a relatively unexplored territory. When studied at low normal loads typically used in tactile exploration of surfaces, studying the mechanisms of friction would help furthering the understanding of importance of friction in tactile exploration of surfaces. Understanding of the role of a finger's surface features on friction can help eliminate unimportant features during the design of skin-like surfaces for robotics and prosthetics applications.

In the present study, dry friction of micro-grit abrasive paper samples was measured for four different probing surfaces: human index finger pad, silicone finger replicas with and without fingerprints, and a smooth silicone sphere. The effect of countersurface and sliding orientation of finger and finger replicas on the coefficient of friction was examined. The role of finger prints, shape of the finger and the elastic properties of finger was investigated and possible friction mechanisms are proposed. 


\section{Materials and Methods}

\section{Materials}

The three samples selected for friction testing were FEPA micro grit abrasive papers with grit sizes of P800, P1200 and P2500 and mean particle sizes of $21.8 \mu \mathrm{m}, 15.3 \mu \mathrm{m}$ and $8.4 \mu \mathrm{m}$ respectively, per manufacturer standards. The corresponding roughness average (Ra) values measured using a contact profilometer (Mahr, MarSurf SD26) were $6.00 \mu \mathrm{m}, 4.23 \mu \mathrm{m}$ and 4.05 $\mu \mathrm{m}$ respectively. Scanning electron microscopy images of the abrasive papers confirmed that the sizes of the particles were in agreement with the particle sizes reported by the manufacturer.

Based on the relative sizes of their particles, the P800, P1200 and P2500 are henceforth referred to as coarse, medium and fine grits, respectively. The abrasive papers were cut into 65 x $20 \mathrm{~mm}$ strips and attached on to a 152 x $101 \mathrm{~mm}$ steel backing plate for testing purposes. Four different types of probe materials were used to test the friction properties: a human finger, a silicone replica of the human finger with finger prints, a silicone replica of the human finger without finger prints and a smooth silicone sphere, as shown in Fig. 1. Silicone is one of the synthetic materials considered to be a reasonable mechanical equivalent to skin on fingers due to similar friction properties $[4,13]$ and the silicone samples were used in the current study to help isolate the effect of fingerprints and the probe (finger) shape on friction. All the tests with the finger were done by using the right hand index finger of one of the researchers. Both the silicone finger replicas with and without fingerprints were modeled after the same finger. Single-use alginate molds (Alja-Safe, Smooth-On Inc.) of the finger were used to cast silicone finger replicas with fingerprints using a platinum cure silicone rubber (Smooth-Sil 950, Smooth-On Inc.). The fingerprints on the human finger were masked and the masked finger was used to make alginate molds for casting silicone finger replicas without fingerprints. The fingerprints were masked by 
applying thin coats of fingernail enamel layer by layer and with intermediate visual inspection to check the degree of masking. This process was used to obtain a finger replica with no fingerprints while retaining the large-scale dimensions and bulk shape of the finger. A dial caliper (Mitutoyo, 505-675) was used to check the consistency of dimensions between the human finger and silicone finger replicas. Twelve silicone replicas each of the finger with and without fingerprints were made. The cured mean durometer hardness of the silicone replicas was 50 Shore A. The silicone sphere (Ultra-strength Silicone Rubber Ball, McMaster-Carr) was $2.54 \mathrm{~cm}$ in diameter (the radius of curvature of the human finger for the contact zone in the current experiment is approximately $1.22 \mathrm{~cm}$ ) and had durometer hardness of 51 Shore A. The mean durometer shore hardness of the human index finger pad was 33 Shore A. All hardness values were measured using an ASTM Type A durometer (PTC Instruments, Model 408). 


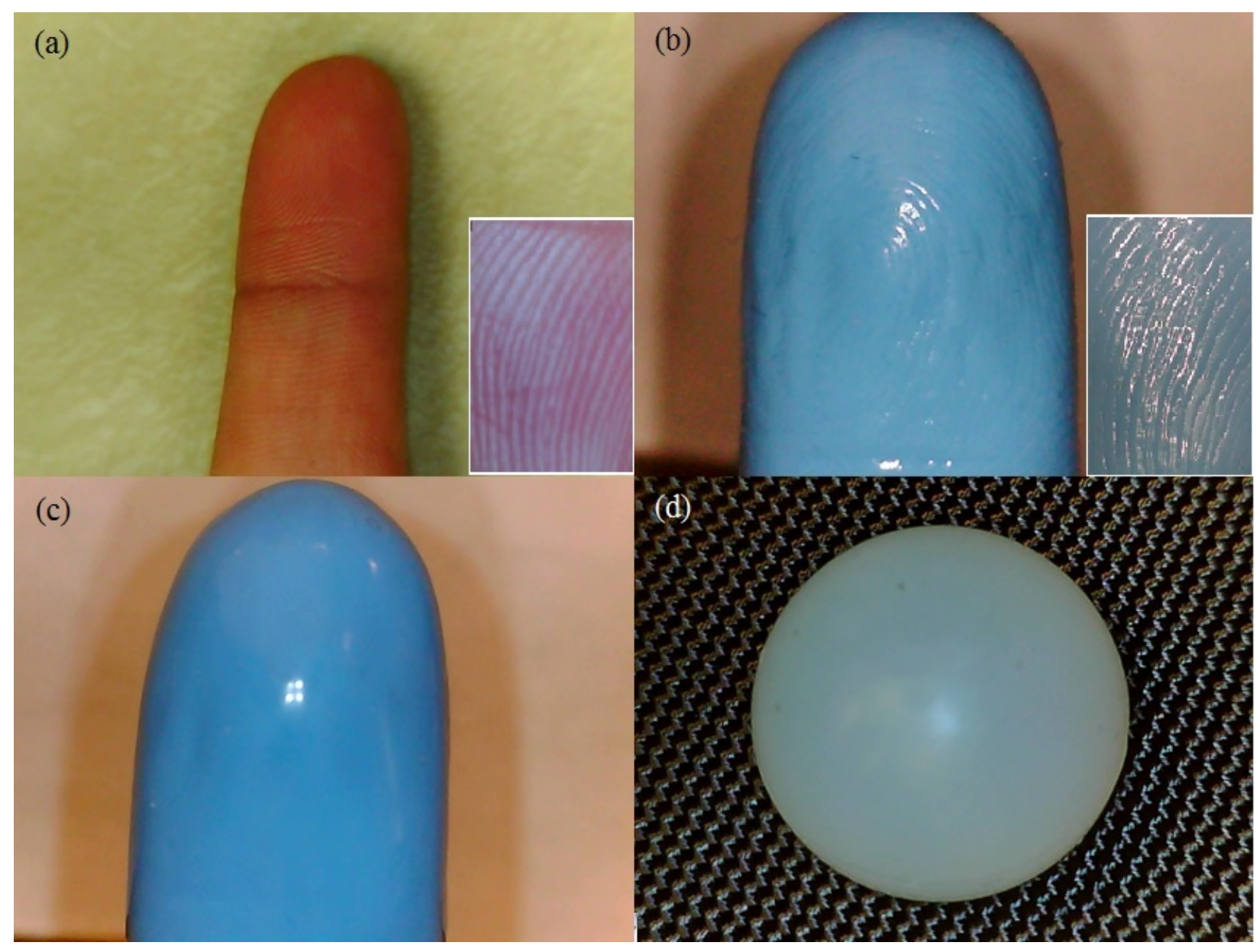

Fig. 1. Probing surfaces used. (a) Right hand index finger, (b) silicone finger replica with finger prints, (c) silicone finger replica without finger prints, and (d) silicone sphere. The inset images in 1(a) and 1(b) show the fingerprint details in the same area of the finger and the silicone finger replica with fingerprints.

\section{Methods}

The surface topography of the abrasive papers was examined using a scanning electron microscope. A FEI Quanta 250 Field emission SEM was used for iridium coated abrasive papers to obtain the images with three dimensional profiles of the abrasive paper surfaces. The SEM images were processed and analyzed using the image analysis software ImageJ.

For the friction property measurement, each of the probes was slid along the length of the abrasive-paper surfaces. The steel backing plates holding the abrasive papers were mounted onto the platform of a three-axis dynamometer (Kistler 9254), which was used to measure the normal 
load applied and the resultant tangential friction force during sliding. During the sliding motion, for the finger and the silicone finger replica samples, the sliding angle with respect to the surface was maintained between $30^{\circ}$ and $45^{\circ}$. The sliding speed was maintained for all the probes between $0.8 \mathrm{~cm} / \mathrm{s}$ and $1.2 \mathrm{~cm} / \mathrm{s}$. A normal load of $0.5 \mathrm{~N}$ with approximate variation of $0.05 \mathrm{~N}$ was applied manually using visual feedback from the dynamometer for all the probes tested. For a spherical surface in contact with a topographically rough flat surface, contact area is linearly proportional to the applied normal load[19]. During the pre-tests, it was noticed that the contact area of the finger and the silicone finger replicas changes with the sliding angle. In effect, the combination of the applied normal load and the sliding angle governs the contact area of the probe, and the alignment of the fingerprint ridges in the contacting area for the ridged probes (human finger and the silicone finger replica with fingerprints). At the applied normal load of 0.5 N, Fig. 2 illustrates the effect of the sliding angle on the contact area of the human finger pad and the corresponding fingerprint ridge orientations. For the higher sliding angles (Fig. 2(a)-2(c)), all the fingerprint ridges were aligned more or less parallel to each other. For lower sliding angles (Fig. 2(d)), the fingerprint ridge alignment was more complex, and as such low angles were not employed in this study of fundamental friction mechanisms.
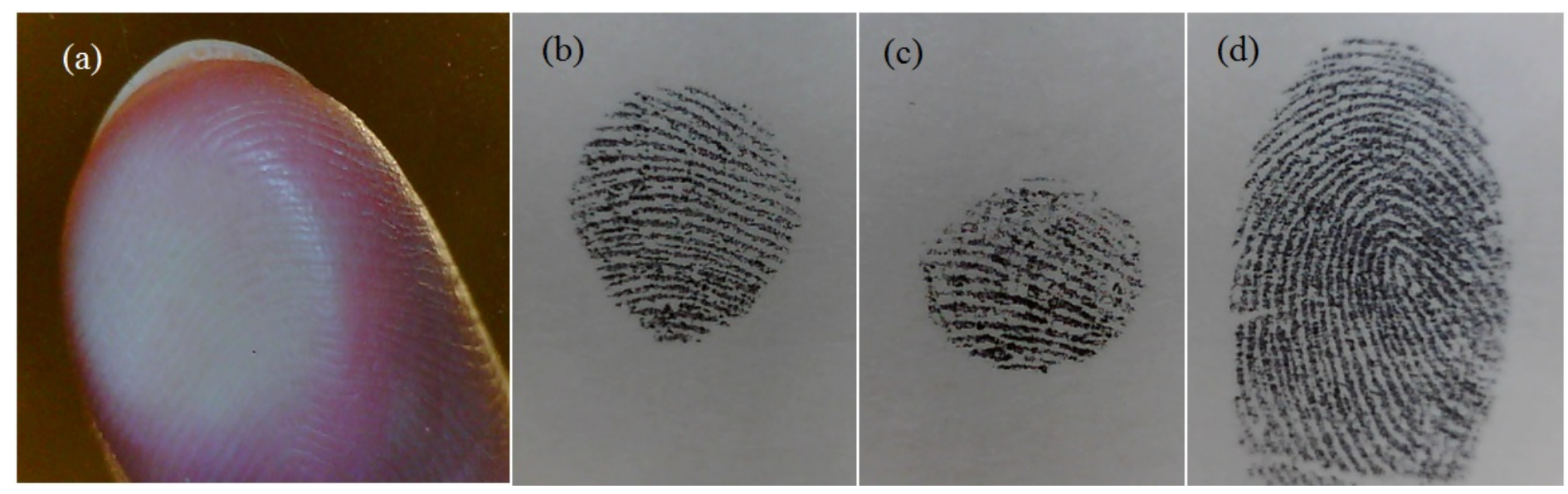
Fig. 2. The contacting areas of different probing surfaces at normal load of $0.5 \mathrm{~N}$. (a) Human finger pad at a sliding angle of approximately $37^{\circ}$. Impressions of human finger pad at sliding angles (b) $\sim 37^{\circ}$, (c) $\sim 85^{\circ}$, (d) $\sim 10^{\circ}$. All the impressions are of same scale. All the images were obtained using a digital microscope (Dino-Lite Basic AM2111).

Based on a preliminary perception experiment done for a previous study, the results of which are presented elsewhere, it was observed by the participants and the researchers that sliding finger across abrasive papers felt different in different orientations of the finger with respect to sliding direction. In order to better understand the nature of this directionality, the friction properties were measured for two sliding orientations (shown in Fig. 3): 1) a 'normal orientation', in which, the fingerprint ridges, for the normal load-sliding angle combination used in the current experiment, were normal / perpendicular to the direction of sliding. The length of the finger was in-line with the direction of the sliding; 2) a 'parallel orientation', in which, the fingerprint ridges were tangential / parallel to the direction of sliding. The length of the finger was perpendicular to the direction of sliding. For the silicone finger replica without fingerprints in spite of the lack of fingerprint ridges, the same orientation convention was used. For the silicone sphere, the surface is both symmetrical and smooth, and as a result, no orientation was assigned for sliding. 


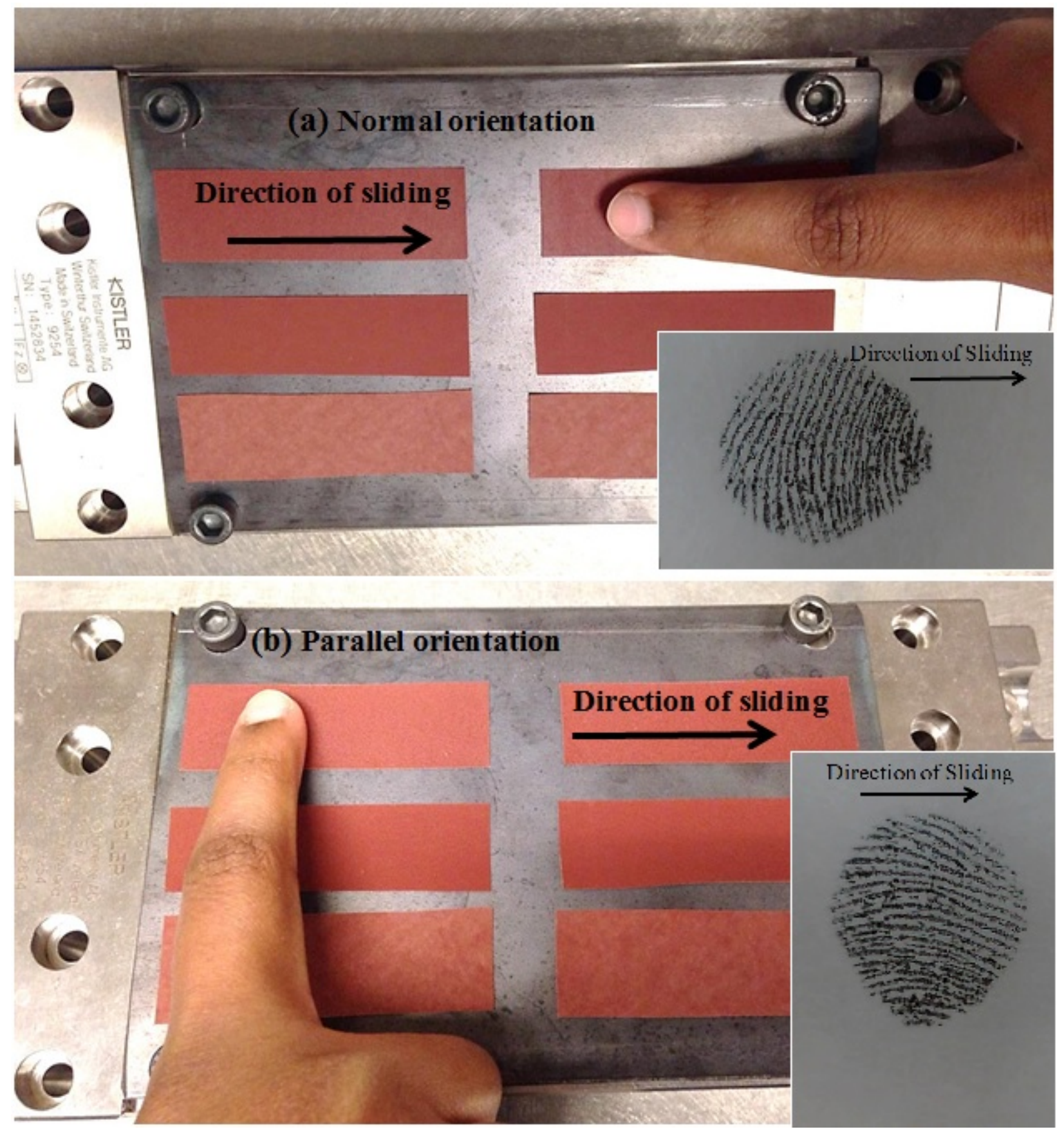

Fig. 3. Sliding orientations used for the friction measurement tests. Finger sliding on P800 (coarse) grit abrasive paper mounted on a Kistler 9254 Dynamometer in (a) normal orientation, (b) parallel orientation. Inset images shows alignment of fingerprint ridges relative to the direction of sliding for the corresponding orientation. Orientations will be similar for the silicone replicas with and without fingerprints.

For the friction measurement, each trial constituted a single swipe across the length (sliding length of approximately $60 \mathrm{~mm}$ ) of the abrasive paper sample with a probe. For each of the 
abrasive paper grits tested, when using a synthetic probing surface (silicone replicas with and without fingerprints and the silicone sphere), 6 trials were performed. Eleven trials were performed for tests with the human finger to account for higher variability. All friction measurements involving a single probe (66 trials for human finger and 36 trials each for rest of the probes), were performed as a single set in a randomized order across abrasive papers and orientations. The random order changed from probe to probe. The normal loads used ( $0.5 \pm .05$ N) were typical of the loads used for tactile exploration [2, 20, 21]. The data acquisition system employed a LabVIEW program for data sampling at $1000 \mathrm{~Hz}$. The coefficient of friction (COF) was calculated using Amonton's law at each time-step for the sampled data. A MATLAB program was employed to find the average coefficient of friction during a trial and, at least 1500 points were considered for computing the average for each trial. A wear particle build up on the surfaces of the probes and the abrasive papers was noticed during preliminary tests when more than 30 trials were performed at once. For finger friction measurements, the hands of the subject were washed and dried thoroughly every 11 measurements to minimize the effect of wear particles deposited during sliding, hydration of skin from sweat, and of oils secreted from the skin. For the silicone finger replicas, each replica was used only for 3 trials (a total of 12 replicas per measurements with each probe), to minimize the effect of the change in topographical features from surface wear and the wear particle build up. For the silicone sphere, the surface in contact was changed every three trials by rotating the sphere. All the friction measurements were performed in a temperature and humidity controlled laboratory setting with a temperature of $23 \pm 2{ }^{\circ} \mathrm{C}$ and relative humidity of $50 \pm 5 \%$. 


\section{Results and Discussion}

\section{Surface microstructure of abrasive papers}

To better understand the friction behavior of the probing surfaces and identify the corresponding underlying friction mechanisms, it is imperative to have an understanding of the topography of the contacting asperities. The SEM images of the abrasive papers were used to study their surface microstructure. The SEM images show that the sizes of the large dimension of the particles were in agreement with the particle size values provided by the manufacturer. The surface of the coarse and the medium abrasive papers consisted of sharp peaked pyramidal abrasive particles aligned mostly perpendicular to the surface of the backing paper with valleylike clear separation between the particles (Fig. 4(a) and 4(b)). Whereas, the surface of the fine abrasive paper had thin plate like abrasive particles aligned more parallel than perpendicular to the surface of the backing paper and overlapping each other (Fig. 4(c)). 

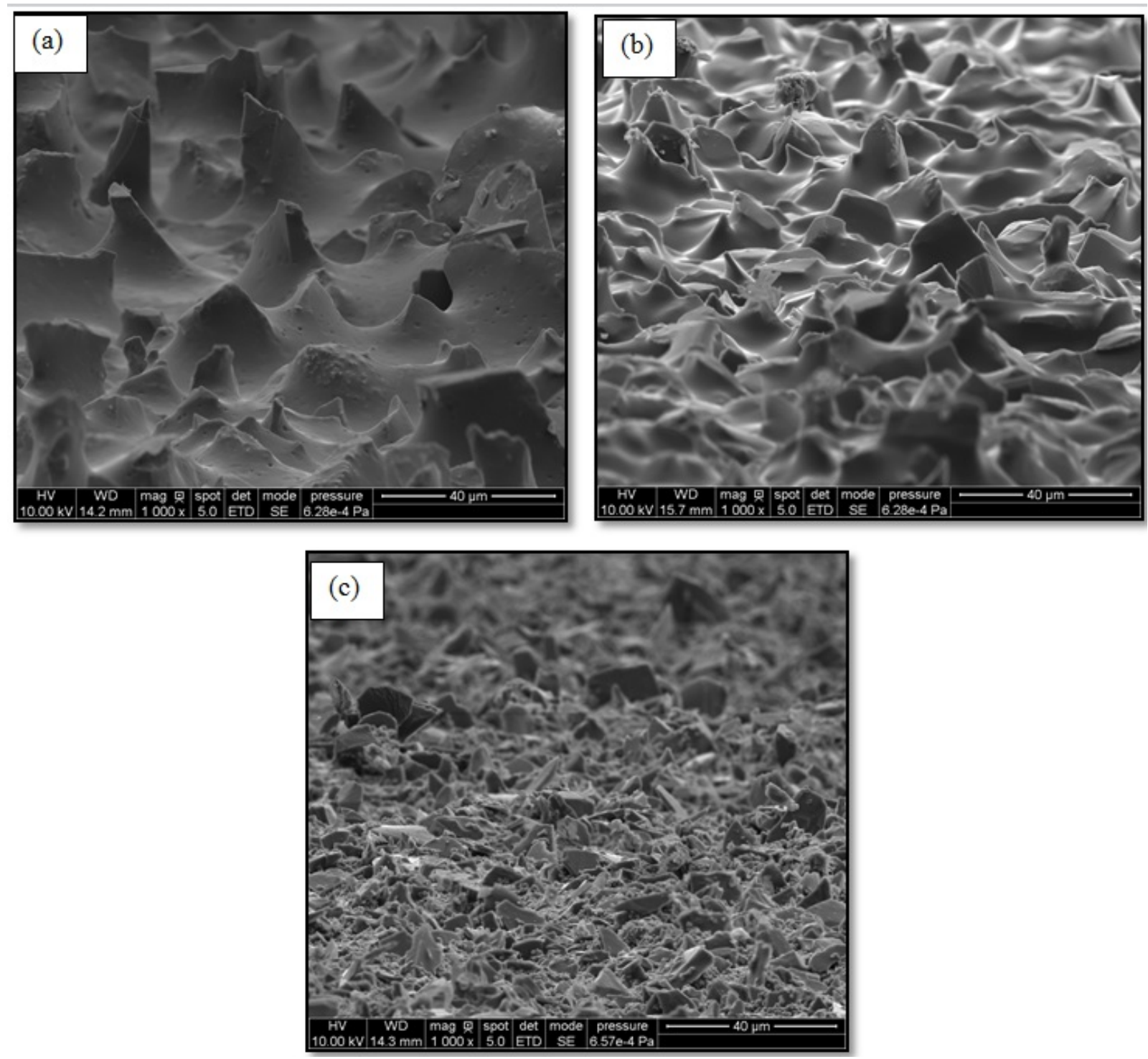

Fig. 4. Scanning Electron Microscope images of abrasive papers (a) P800 (coarse) (b) P1200 (medium) (c) P2500 (fine). All images were secondary electron images at $1000 \mathrm{X}$ and $85^{\circ}$ tilt. The horizontal scale bar is $40 \mu \mathrm{m}$ in length.

\section{Trends in the coefficient of friction}

The coefficients of friction of the three abrasive papers, coarse, medium and fine, were measured against the four different probes in the two different sliding orientations (normal and parallel to the fingertip or simulant ridges). This was done to understand the effect of the shape, material properties and the presence of fingerprint ridges on friction during tactile exploration with texture elements smaller than the size of the ridges. The COF measurements from the sliding 
tests on abrasive papers with different probing surfaces were plotted as a function of the mean particle size of the abrasive papers to evaluate the trends in the coefficients of friction (Figs. 5 and 7). The mean particle size was a defining variable that differentiates different abrasive paper grits, and was used for this reason as the independent variable for plotting COF values. The value of the mean particle size increased linearly from the fine to the coarse abrasive papers. Due to its symmetrical and featureless smooth surface, the friction properties of the silicone sphere did not have orientation effects, and the COF values for the silicone sphere were represented in plots for both the normal and parallel orientations.

The coefficient of friction of the silicone sphere decreased from the fine to the medium grits and then increased from the medium to the coarse grits. In the normal orientation of the human finger and the silicone finger probes with and without fingerprints, the same trend in COF values was observed. Also, in the parallel orientation of the silicone finger probe without fingerprints, the trend in COF from finest to the coarsest grit was the same. The amount of decrease from the fine to the medium and the amount of increase from the medium to the coarse grits was different for different probes. However, the decrease was dramatic for the human finger pad in the normal orientation. Also, for the human finger pad in the parallel orientation, the COF increased from the fine through coarse grits and, for the silicone finger with finger prints the COF values increased from the fine to medium grits and then changed little (Fig. 8). The possible reasons for such orientation dependent effects and the frictions mechanisms responsible are discussed in detail in the subsequent sections. 

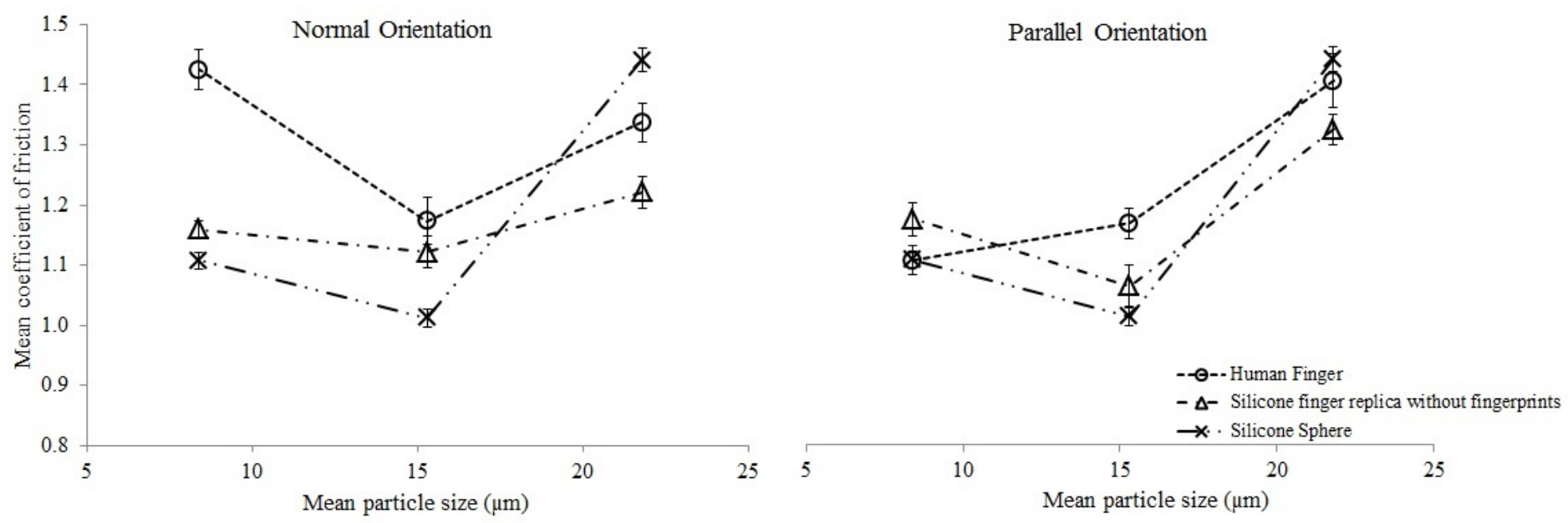

Fig. 5. Variation of coefficient of friction of the abrasive papers for the human finger, the silicone finger replica without fingerprints and the silicone sphere as a function of the mean particle size of the abrasive papers in normal orientation and parallel orientations. Error bars indicate standard error of the mean coefficient of friction. The mean particle size of the fine, medium and the coarse grits are $8.4 \mu \mathrm{m}, 15.3 \mu \mathrm{m}$ and $21.8 \mu \mathrm{m}$ respectively

\section{Friction Mechanisms}

The net friction between the skin and a contacting surface has been suggested to be a summation of two terms: 1) adhesive friction and, 2) a deformation friction [22]. The adhesion friction results from breaking temporary bonds formed between the surface atoms of the interacting surfaces[23] and in the case of skin friction, adhesion coefficient of friction $\mu_{a}$ could be expressed by the equation:

$$
\mu_{a}=\frac{\tau_{0} A}{N}+\alpha
$$

where $\tau_{0}$ is the interfacial shear strength, $A$ is the real contact area between the interacting surfaces, $N$ is the applied normal load and $\alpha$ is the pressure coefficient[22]. The equation suggests that the change in real contact area causes change in adhesion component of friction.

The deformation friction results from the imbalance of surface contact around an asperity due to the partial recovery (hysteresis) of the viscoelastic material, which is deformed by the rigid 
asperities of the interacting surface, and for a rigid conical slider interacting with an elastomeric surface, the deformation coefficient of friction due to hysteresis $\mu_{h}$ could be obtained from the equation derived by Greenwood and Tabor[24]:

$$
\mu_{h}=\frac{2\left(1-\sigma^{2}\right)}{\pi E} \bar{p} \beta
$$

where $\beta$ is the hysteresis loss fraction, $\bar{p}$ is the mean pressure defined as ratio of the normal and contact area, $\sigma$ and $\mathrm{E}$ are the Poisson's ratio and elastic modulus respectively of the elastomer. It can be observed from this equation that the increase in the contact pressure or the decrease in elastic modulus could increase the deformation component of friction.

The repetitive motion of fingerpad on abrasive paper surfaces could result in scratching of the finger surface and abrasive wear. Deformation friction in such a scenario was termed as 'ploughing friction' by Derler et al. and the ploughing coefficient of friction $\mu_{p}$ obtained by modelling skin as an elastomeric surface in contact with a rigid conical sliders was given by the equation:

$$
\mu_{p}=\frac{2}{\pi} \tan \phi
$$

where $\phi$ is the mean slope of the conical sliders with the interface[15]. The ploughing friction clearly depends on the slope of the hard sliders with the softer interface and it can be said that shaper the indenter higher is the deformation component of friction.

In addition, a third interlocking term has been proposed to model the friction force for skin in contact with ridged surfaces. Interlocking friction arises from the resistance encountered by the 
ridges of the finger climbing over the asperities of the interacting surface and was estimated the interlocking coefficient of friction $\mu_{i}$ for a triangular ridged surface in contact with finger pad as:

$$
\mu_{i}=\cot \delta
$$

where $\delta$ was the angle between triangular ridge and the vertical center line[7]. From the equation, it can be said that sharper the ridged surface greater is the interlocking component. The same could be applied for the abrasive particles sliding over the surface of the fingerpad. .

The silicone sphere had a nominally smooth featureless surface such that the friction properties were likely due to only adhesion and deformation mechanisms. The fine grit abrasive paper's surface topography consisted of flat closely aligned particles. This could lead to high real contact area when interacting with smooth surface like that of the silicone sphere, and consequently, a high adhesion component of friction. However, due to the same flat particle the deformation component of friction could be quite low. So, the net friction could be dominantly due to adhesion. For the medium and the coarse grits, with sharp peak-like particles, the adhesion between the abrasive papers and the silicone sphere could decrease due to decrease in the real contact area. However, the deformation friction could potentially increase due to the increase in the sharpness of the contacting asperities. The resultant coefficient of friction would be a combination of these two effects. The deformation component of friction could increase with the increase in size of the asperities. This could result in a higher deformation friction for the coarse grit compared to medium grit. From the COF data for the silicone sphere it was observed that, the COF for the fine grit was high, followed by a decrease in the COF leading to a minimum for the medium grit and then an increase with the increasing asperity size reaching a maximum for the coarse grit. Based on this COF trend, it would be safe to reason that adhesion was the 
dominant mechanism for the fine grit, contributions from both adhesion and deformation components were reasonably important for the medium grit and deformation was the dominant mechanism for the coarse grit.

From the friction data it can also be observed that the COF for the silicone sphere for the coarse grit was the highest, and for the medium and the fine grits was the lowest compared to corresponding coefficients of frictions for the other probing surfaces and the same frictional trend could be seen in both the sliding orientations. This trend in COF for the silicone sphere combined with its symmetrical structure and a lack of identifiable surface features makes it ideal to be used as a baseline comparison for the other probes to understand the effects of their respective topography and material properties on the dominant friction mechanisms.

\section{Effect of shape of the finger}

Modifying the shape of the probe from silicone sphere to finger could change the contact area, whose positive changes lead to an increase in the adhesion component of friction and a decrease in the deformation component of friction. Depending on the dominating friction mechanism for a given interacting surface, the COF could change accordingly. The coefficients of friction of the silicone finger replica without fingerprints were compared with those of the silicone sphere to understand this effect (Fig. 5).

The contact surface for the silicone sphere was a circle (Fig. 6(a)), and that of the silicone finger replica was approximately an ellipse and (Fig. 6(b)) with its minor axis similar to the radius of the circle. There was a measured increase in the contact area for silicone finger replica without fingerprints compared to the silicone sphere. The increase in the contact area could lead to an

increase in the adhesion component of friction. The deformation component of friction could 
decrease due to a decrease in the contact pressure. When compared with the silicone sphere, the net result would be an increase in the COF for the fine grit for which adhesion was the dominant mechanism and a decrease in the COF for the coarse grit for which deformation was the dominant mechanism. That was indeed the case with experimental COF values for the fine and coarse grits. For the medium grit, no meaningful prediction could be made without knowing the relative contributions of adhesion and deformation components to the friction. However, the experimental COF values suggest that adhesion friction could be contributing relatively more than the deformation component, as witnessed by the overall increase in the COF for the medium grit.

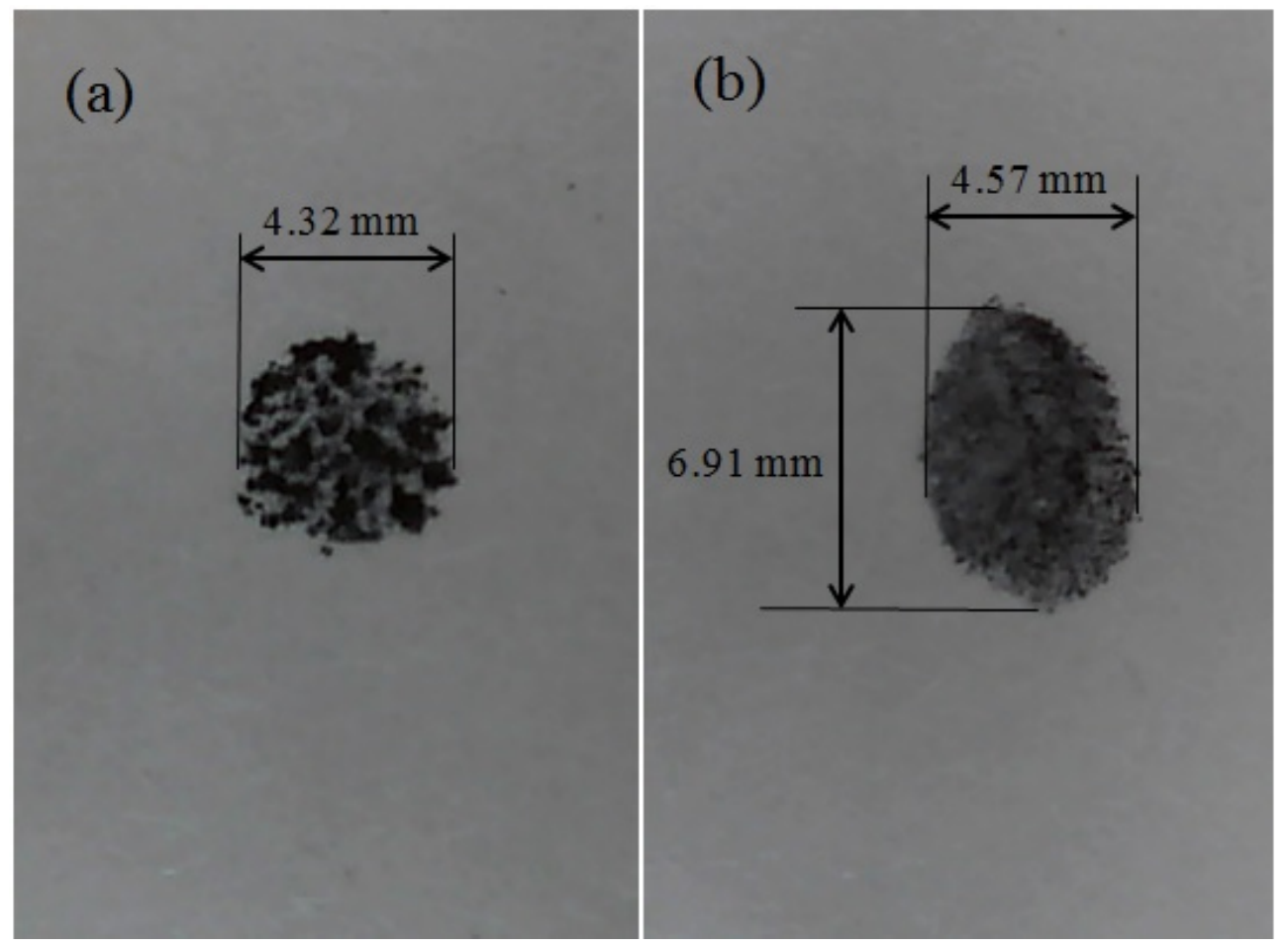

Fig. 6. (a) Contacting area of the silicone sphere. (b) Contacting area of the silicone finger replica without fingerprints at a sliding angle of $\sim 30^{\circ}$. All Impressions were scanned using a digital microscope (Dino-Lite Basic AM2111) and were measured using Image J image analysis software. 


\section{Effect of the fingerprints}

The presence of fingerprint ridges could add an interlocking contribution to the coefficient of friction, and a possible decrease in the adhesion and deformation components. However, the effect could be complex and vary with multiple factors like the shape and size of the contacting asperities, sliding direction of the finger and the angle of sliding. The effect of fingerprints was examined by comparing silicone finger replicas with and without fingerprints (Fig. 7).

For the silicone replica with fingerprints, when compared with the silicone replica without fingerprints, the presence of fingerprint grooves could decrease the real contact area, decreasing the adhesion component of the coefficient of friction. Also, the presence of grooves could decrease the penetration of asperity into the silicone finger surface in the groove space, resulting in a decrease in the deformation component of friction. Decrease in both of these components depends on the width of the fingerprint groove. Further, for the silicone replicas with fingerprints, interlocking could take place when the asperities of the abrasive papers pass from inside the grooves of the fingerprints over to the top of the ridges of the fingerprints. The contribution of interlocking depends on the relative sizes of depth of the fingerprint groove and the height of the asperities. The alignment of the fingerprint ridges in the contacting area combined with the sliding direction determines the zone in which interlocking can happen. At the sliding angles and the normal load employed in the current study, the fingerprint ridges in the contacting area were all aligned and oriented in the same direction, and the interlocking effect was possible only in the normal orientation. 

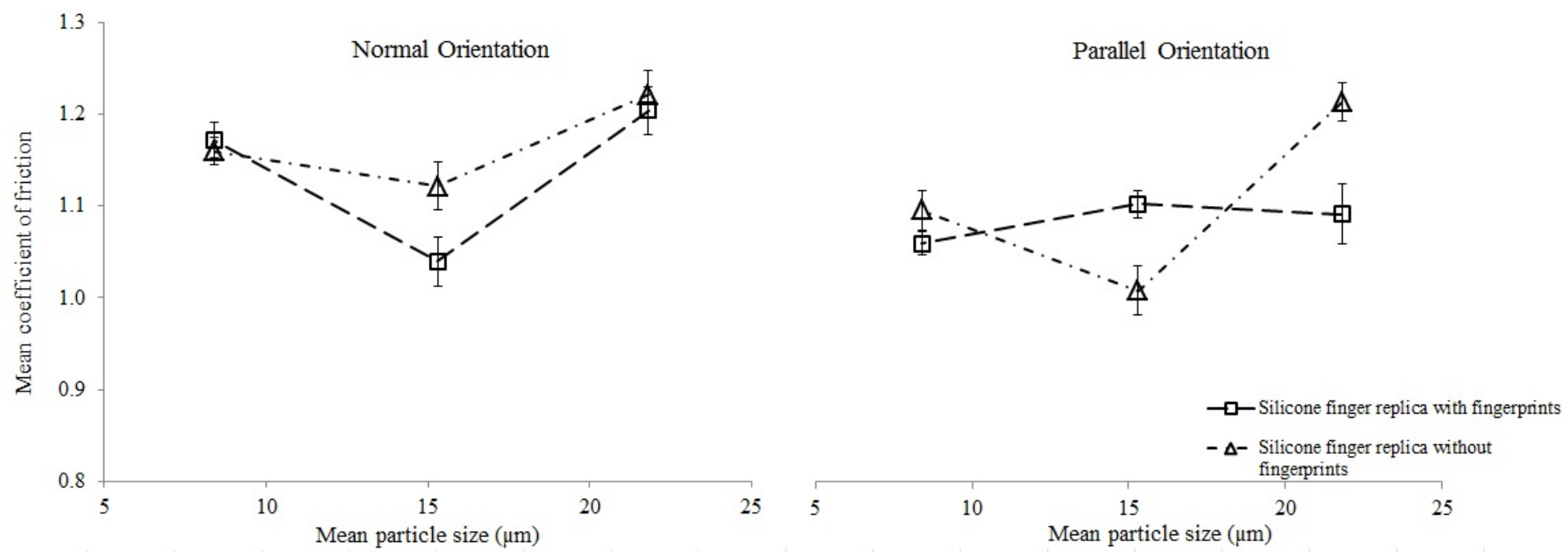

Fig. 7. Variation of coefficient of friction of the abrasive papers for the silicone finger replicas with and without fingerprints as a function of the mean particle size of the abrasive papers in normal and parallel orientations. Error bars indicate standard error of the mean coefficient of friction.

In the normal orientation of the silicone replicas with fingerprints, the fingerprint ridges were perpendicular to the direction of motion of the finger (Fig. 3(a)) and the interlocking was possible during relative motion of fingerprint ridges over the surface asperities of the abrasive papers. The effects of interlocking however depend on the relative sizes of contacting asperities[7]. For the human finger, the ridge width and groove widths are approximately $400 \mu \mathrm{m}$ and $150 \mu \mathrm{m}$ respectively [9] and the depth of the groove was approximately $25 \mu \mathrm{m}$ [17]. For the fine grit, with very small flat particles (mean particle size $8.4 \mu \mathrm{m}$ ) aligned almost parallel to its surface, the increase in COF from interlocking would be small and there could be a slight decrease in the adhesion component of friction due to decrease in real contact area in the groove space. The COF would be more or less the same for the replicas with fingerprints and the replicas without fingerprints. This was in agreement with the experimental values of COF. For the medium grit (mean particle size $15.3 \mu \mathrm{m}$ ), interlocking could take place. The deformation component of friction could decrease due to a decreased penetration of asperities in the groove space and the adhesion component of friction due to loss of contact in the groove space. The 
COF however would depend on the relative contribution of interlocking compared to the other two components. The decrease in the experimental coefficient of friction value of the silicone replicas with fingerprints from that the silicone replicas without fingerprints suggests that the asperities were still small enough for any considerable interlocking effects to take place. For the coarse grit (mean particle size $21.8 \mu \mathrm{m}$ ), the size of asperity might be close enough to the depth of the fingerprint grooves to create significant interlocking effects. However, the deformation component of friction could decrease due to lack of deformation in the groove space. The net effect would depend on the whether contribution of interlocking was sufficient to offset the decrease in deformation component. There was no change in the experimental value of COF from silicone replicas without fingerprints to the replicas with fingerprints, suggesting that interlocking was indeed possible.

For the parallel orientation, the fingerprint ridges were parallel to the direction of motion of the finger (Fig. 1(b)) and the effect of fingerprints was a slight decrease in COF for the fine grit, an increase for the medium grit and a dramatic decrease for the coarse grit. Very little interlocking effect was assumed to be possible as the relative motion of the asperities of the abrasive papers was either along the edge of the ridge, in the grove or on top of the ridge. Based on the fingerprint ridge and groove dimensions, the size of the abrasive paper asperities and spacing between abrasive paper asperities, the following are possible explanations from the trends in the coefficient of friction. For the fine grit, considering the relative sizes of asperities and the depth of the fingerprint groove, the asperities that lie in the fingerprint groove could have no contact with the groove surface. The additional contact area from the asperity contact along the edge of the ridge was negligible due to flat plate like nature of the fine grit's abrasive articles (Fig. 4(c)). This could result in a decrease in the adhesion friction due to the decrease in the real contact area 
and in turn the decrease in the COF. For the medium grit, the adhesion component of the friction could increase from the increase in the number of asperity surfaces in contact along the edges of the ridges. From the asperities moving in the grooves, there could be no possible contribution to the deformation of the probe surface and could result in a decreased deformation component of the friction. The net effect however would depend on relative contributions of adhesion and deformation components to the friction. The increase in the experimental COF supports that previous observation about the adhesion friction possibly contributing relatively more than the deformation component for the medium grit. For the coarse grit, due to its larger asperities, the loss of deformation could be higher from the asperities moving in the grove. With the deformation hypothesized to be the dominant contributor to the friction, the effect of decrease in deformation friction could be more dramatic on the net friction in comparison with the effect of increase in adhesion friction. The decrease in the coefficient of friction from silicone replicas without fingerprints to that of the replicas with fingerprints for the coarse grit supports the hypothesis.

\section{Effect of the material properties in combination with the fingerprints}

The effect of material properties in combination with the fingerprints was analyzed by comparing the human finger pad friction values with those of the silicone replica with fingerprints (Fig. 8). The effect of change in material properties from silicone replica to a finger was a marked increase in all the three components of friction. However, there were some possible interaction effects between the interlocking and adhesion mechanisms, as well as between the interlocking and deformation mechanisms.

Elastic modulus of the probing surfaces can be roughly estimated using their durometer shore hardness values using Gent's semi empirical relation[25]. The durometer shore hardness of the 
human finger pad (32 Shore A) was less than that of the silicone replicas with fingerprints (50 Shore A) and from Gent's relation, the elastic modulus of the finger was less than that of the silicone samples. The lower elastic modulus could enable the surface of the skin to conform itself around the countersurface asperities more than the silicone. This could increase the adhesion component of friction due to increased real contact area and the interlocking component of the friction whenever interlocking was possible. That is, wherever interlocking was possible, for a given normal load, finger-on-asperities had higher probability of interlocking to take place than the silicone replica with fingerprints. Also, when interacting with countersurface asperities of given hardness (greater than that of human skin or silicone allowing their deformation), localized deformation for finger pad could more due to its lower shore hardness than that of the silicone replica. This could increase the deformation component of friction.
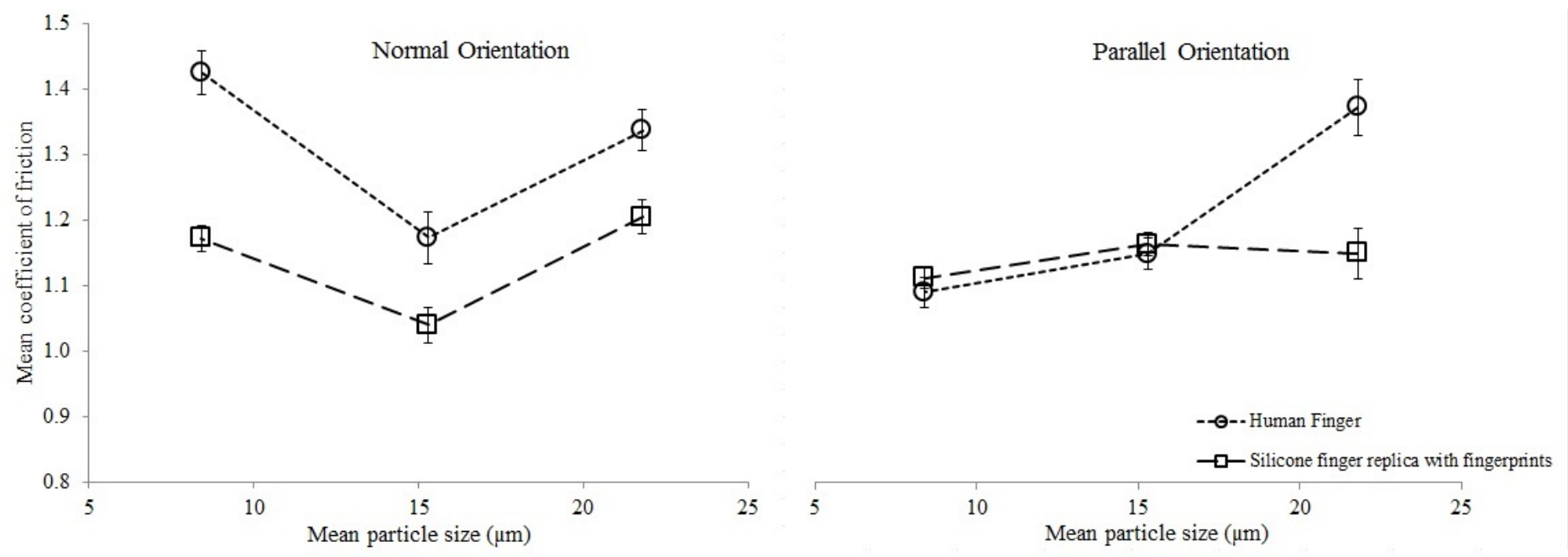

Fig. 8. Variation of coefficient of friction of the abrasive papers for the human finger and the silicone finger replica with fingerprints as a function of the mean particle size of the abrasive papers in normal and parallel orientations. Error bars indicate standard error of the mean coefficient of friction.

For the normal orientation, there was a possibility of interlocking to happen for both the human finger and the silicone replica. For all the three abrasive paper grits, there was an increase in the mean coefficients of friction of human finger compared to those of the silicone replica with 
fingerprints. Based on the hypothesized dominant friction mechanisms for each of the grits, for the fine grit, the increase could be due to a combination of the increase in adhesion and interlocking components of friction. It is believed that for the medium grit, the increase could from an increase in the interlocking component and for the coarse grit, the increase could be from a combination of the interlocking and deformation friction components. However, for the coarse grit the increase in the coefficient of friction in the normal orientation was less marked than that in the parallel orientation. It was possible for the interlocking action to create harder zone ahead of it leading to a decrease in the amount of deformation and a consequent decrease in the deformation component of the friction.

For the parallel orientation, no interlocking effect was possible for either of the probes. There was little change in the coefficients of friction of the fine and the medium grits for human finger when compared to those for the silicone replica with fingerprints. This suggests that interlocking component could indeed have been responsible for increase in the coefficients of friction for the fine and the medium grits. However, the possible increase in adhesion friction component for the fine grit was more pronounced in the normal orientation and was not as evident in the parallel direction. One probable explanation was bending of fingerprint ridges in the direction of motion of the finger as the as the asperities of the abrasive paper move over them in the normal orientation. The bending could have caused an increased contact area and a consequent increase in the adhesion friction. Due to the lower elastic modulus of the finger, this effect would likely be more pronounced than for the silicone replica. For the coarse grit, the increase in COF could be from an increase in the deformation friction. 


\section{Conclusions}

In this study, coefficients of friction of micro-grit abrasive papers for index finger pad and different silicone surfaces were measured at normal loads typically used for tactile exploration. The goal was to isolate the effects of fingerprints, shape and material properties of the finger and the finger replicant probes. Possible friction mechanisms involved were discussed. The following conclusions can be drawn from the results of this study.

- The fingertip coefficient of friction when interacting with textured surfaces of small elements $(<50 \mu \mathrm{m})$ showed strong evidence of obeying a summation of adhesion, deformation and interlocking components.

- The shape of the finger was responsible for an increase in contacting surface area, which further affected the adhesion and deformation components of friction. Thus, probe shape appeared to have a significant impact on the overall coefficient of friction.

- The presence of fingerprint ridges is hypothesized to add an interlocking contribution to the overall coefficient of friction, based on the results. The contribution of interlocking friction can vary with multiple factors like the shape and size of the contacting asperities, sliding direction of the finger and the angle of sliding. Further, the fingerprint ridges appeared to cause a decrease in the deformation and the adhesion components of friction.

\section{Acknowledgements}

The authors greatly appreciate the financial support for this work. This material is based upon work supported by the National Science Foundation under Grant No. 1262797 


\section{References}

[1] Westling, G., Johansson, R. Factors influencing the force control during precision grip. Exp Brain Res 53:277-284 (1984).

[2] Smith, A., Gosselin, G., Houde, B. Deployment of fingertip forces in tactile exploration. Exp Brain Res 147:209-218 (2002).

[3] Lewis, R., Menardi, C., Yoxall, A., Langley, J. Finger friction: Grip and opening packaging. Wear 263:1124-1132 (2007).

[4] Derler, S., Schrade, U., Gerhardt, L.C. Tribology of human skin and mechanical skin equivalents in contact with textiles. Wear 263:1112-1116 (2007).

[5] Darden, M.A., Schwartz, C.J. Investigation of skin tribology and its effects on the tactile attributes of polymer fabrics. Wear 267:1289-1294 (2009).

[6] Tomlinson, S.E., Lewis, R., Carré, M.J. The effect of normal force and roughness on friction in human finger contact. Wear 267:1311-1318 (2009).

[7] Tomlinson, S.E., Carré, M.J., Lewis, R., Franklin, S.E. Human finger contact with small, triangular ridged surfaces. Wear 271:2346-2353 (2011).

[8] Tomlinson, S., Lewis, R., Liu, X., Texier, C., Carré, M. Understanding the Friction Mechanisms Between the Human Finger and Flat Contacting Surfaces in Moist Conditions. Tribol Lett 41:283-294 (2011).

[9] Derler, S., Gerhardt, L.C., Lenz, A., Bertaux, E., Hadad, M. Friction of human skin against smooth and rough glass as a function of the contact pressure. Tribology International 42:15651574 (2009).

[10] Pasumarty, S., Johnson, S., Watson, S., Adams, M. Friction of the Human Finger Pad: Influence of Moisture, Occlusion and Velocity. Tribol Lett 44:117-137 (2011).

[11] Tomlinson, S.E., Lewis, R., Carré, M.J., Franklin, S.E. Human finger friction in contacts with ridged surfaces. Wear 301:330-337 (2013).

[12] Shao, F., Childs, T.H.C., Henson, B. Developing an artificial fingertip with human friction properties. Tribology International 42:1575-1581 (2009).

[13] Hyun-Yong, H., Shimada, A., Kawamura, S.: Analysis of friction on human fingers and design of artificial fingers. pp. 3061-3066. Place (1996)

[14] Masen, M.A. A systems based experimental approach to tactile friction. Journal of the mechanical behavior of biomedical materials 4:1620-1626 (2011).

[15] Derler, S., Preiswerk, M., Rotaru, G.M., Kaiser, J.P., Rossi, R.M. Friction mechanisms and abrasion of the human finger pad in contact with rough surfaces. Tribology International 89:119127 (2015).

[16] Prevost, A., Scheibert, J., Debrégeas, G. Effect of fingerprints orientation on skin vibrations during tactile exploration of textured surfaces. Communicative \& integrative biology 2:422 (2009).

[17] Scheibert, J., Leurent, S., Prevost, A., Debrégeas, G. The role of fingerprints in the coding of tactile information probed with a biomimetic sensor. Science (New York, NY) 323:1503 (2009).

[18] Wandersman, E., Candelier, R., Debrégeas, G., Prevost, A. Texture- induced modulations of friction force: the fingerprint effect. Physical review letters 107:164301 (2011).

[19] Adams, M.J., Johnson, S.A., Lefèvre, P., Lévesque, V., Hayward, V., André, T., et al. Finger pad friction and its role in grip and touch. Journal of The Royal Society Interface 10:20120467 (2013). 
[20] Smith, A.M., Scott, S.H. Subjective scaling of smooth surface friction. Journal of neurophysiology 75:1957 (1996).

[21] Smith, A., Chapman, C., Deslandes, M., Langlais, J.-S., Thibodeau, M.-P. Role of friction and tangential force variation in the subjective scaling of tactile roughness. Exp Brain Res 144:211-223 (2002).

[22] Adams, M., Briscoe, B., Johnson, S. Friction and lubrication of human skin. Tribol Lett 26:239-253 (2007).

[23] Stachowiak, G.W.: Engineering Tribology. Butterworth-Heinemann, (2006)

[24] Greenwood, J.A., Tabor, D. The Friction of Hard Sliders on Lubricated Rubber: The Importance of Deformation Losses. Rubber Chemistry and Technology 33:129-141 (1960).

[25] Gent, A.N. On the Relation between Indentation Hardness and Young's Modulus. Rubber Chemistry and Technology 31:896-906 (1958). 